\title{
Interference Cancellation Scheme for Three-hop Cooperative Relay Networks
}

\author{
Yinghua Zhang ${ }^{1,2}$, Lei Wang1, Jian Liu ${ }^{1, *}$ and Yunfeng Peng ${ }^{1}$ \\ ${ }^{1}$ School of Computer and Communication Engineering, University of Science and Technology Beijing, \\ Beijing 100083, China \\ [e-mail: 82774807@qq.com; ustb_wl16@163.com; liujian@ustb.edu.cn; pengyf@ustb.edu.cn] \\ ${ }^{2}$ Dawning Information Industry (Beijing) Corp., Ltd, Beijing 100193, China \\ [e-mail: zhangyh@sugon.com] \\ *Corresponding author: Jian Liu
}

Received November 24, 2018; revised March 9, 2019; accepted March 25, 2019; published September 30, 2019

\begin{abstract}
In this paper, we focus on interference cancellation for three-hop cognitive radio networks (CRNs) over Rayleigh fading channels. In CRNs, secondary users (SUs) are allowed to opportunistically utilize the licensed spectrum during the idle time of primary users (PUs) to achieve spectrum sharing. However, the SUs maybe power constrained to avoid interference and cover a very short transmission range. We here propose an interference cancellation scheme (ICS) for three-hop CRNs to prolong the transmission range of SUs and improve their transmission efficiency. In the proposed scheme, a flexible transmission protocol is adopted to cancel the interference at both secondary relays and destinations at the same time. And a closed-form expression for the secondary outage probability over Rayleigh fading channels is derived to measure the system performance. Simulation results show that the proposed scheme can significantly reduce the secondary outage probability and increase the secondary diversity in comparison with the traditional cases.
\end{abstract}

Keywords: Cognitive radio networks, interference cancellation, power control, secondary outage probability

This work is supported by National Major Project (No. 2017ZX03001021-005), and also supported by the National Key R\&D Program of China (2017YFB1001600). 


\section{Introduction}

In the past years, the development of the fifth generation (5G) mobile communications has captured the attention of researchers around world [1][2]. One of the evolving directions of 5G is to decrease the size of cells, which will significantly improve the cell throughput, energy efficiency and user data rate of cellular system [3]. Dense network deployment exhibits the above advantages, but produces a series of problems such as co-channel interference [4]. Interference becomes a major limiting factor for higher data rates [5]. Many new schemes have been proposed for interference cancellation [6][7].

With the development of $5 \mathrm{G}$, cognitive radio (CR) technology is promising in improving spectral efficiency by allowing secondary users (SUs) to opportunistically utilize the licensed spectrum owned by primary users (PUs) [8]-[10]. Although licensed bands are heavily exploited, it has been shown that some licensed bands are underutilized. Cognitive radio allows SUs to dynamically utilize the underutilized licensed bands without causing harmful interference to PUs [11]-[13]. Generally, SUs operate in three kinds of paradigms, called interweave, overlay, and underlay, to exploit the licensed spectrum. Various aspects related to the spectrum sharing pattern have been addressed [14]-[16]. In [17], a transmission protocol has been proposed to enable SUs to utilize the licensed spectrum detected as unoccupied. The interweave spectrum sharing paradigm appears to be more sensitive to the spectrum sensing errors, which leads to low secondary transmission efficiency [18]. To overcome the disadvantages of interweave spectrum sharing, underlay spectrum sharing has been adopted, which permits SUs to utilize the licensed spectrum regardless of PU traffic patterns [19]. Different from the interweave paradigm, SUs transmit signals as long as the interference to PUs does not exceed a given threshold. The secondary transmission performance of two-hop decode-and-forward (DF) relaying under the underlay paradigm is measured by the secondary outage probability in [20]. However, there are still several problems to be solved due to the limited secondary transmission power. For example, the effective distance in environments with high path loss should be reduced and the transmission coverage area should be expanded [21].

As a result, several studies have been carried out to use multi-hop relays to enhance the performance of CRNs [22][23]. Multi-hop communication systems effectively extend the transmission range and increase the quality of service (QoS) of relay networks [24][25].

However, with the increase of the number of relay hops, the quality of signal transmission may be reduced [26][27]. Many conventional transmission protocols, such as interference cancellation (IC), relay selection, and data equalization, have been utilized in traditional CRNs to reduce the interference of primary users and improve the secondary transmission performance [28]-[30]. The IC-based underlay CRNs are considered in [17] and [20], where the single-hop and two-hop transmissions are studied. In [31], the author derives a cooperative outage probability model and a cooperative block error rate (BLER) model to mitigate co-channel interference and improve the system performance. Recently, [32] investigates an interference cancellation method when there are two interferences simultaneously, in which a system model was established to analyze the interference cancellation performance.

Different from [17] and [20], we propose an IC-based three-hop cognitive transmission scheme, which is more flexible for the underlay paradigm. This three-hop model is the simplest and most typical model for multi-hop transmission. Taking into account the actual signal transmission range and direct transmission distance in the cell, we investigate the 
performance of a cognitive radio system, consisting of a single secondary transmitter (ST), a single secondary destination (SD), and two relay clusters, i.e., three hops that assist the ST to transmit signals to the SD. Our main contributions can be summarized in two-folds.

In this paper, we expand the secondary transmission range and improve the secondary transmission performance. Specifically, we use a simplified system model and utilize the secondary outage probability to measure the performance of the secondary system. Under the strict power constraints, we propose a three-phase interference cancellation scheme (ICS), which can expand the transmission range and improve the performance of the secondary system. The ICS aims at promoting the QoS of secondary transmission measured by secondary outage probability. Note that our scheme enables more flexible relay selection with lower secondary outage probability in comparison with traditional cases [17] [20].

Based on the interpretation of ICS for underlay communication system, we derive the closed-form expression for outage probability over Rayleigh fading channels. Then we conduct the simulations and demonstrate the superiority of the performance analysis by using Monte Carlo methods. The improved system achieves better secondary performance than that of [20] due to the flexible protocol.

The remainder of this paper is organized as follows: In section 2, we present an overview of the system model and propose the protocol for three-hop relay system in this paper, while in section 3 we provide the analytical results on the performance in terms of secondary outage probability and then derive the closed-form expressions of the system performance. In section 4, we provide the numerical and simulation results, while in section 5 we draw the conclusions.

\section{System Protocol}

We consider a cognitive radio system with the coexistence of primary and secondary networks, as shown in Fig. 1. In the primary networks, primary transmitter PT delivers signals to primary destination PD. In the secondary network, a secondary transmitter ST forwards its signal towards secondary destination SD, while a route of clusters $S R_{i}$ assisting ST data transmission to SD.

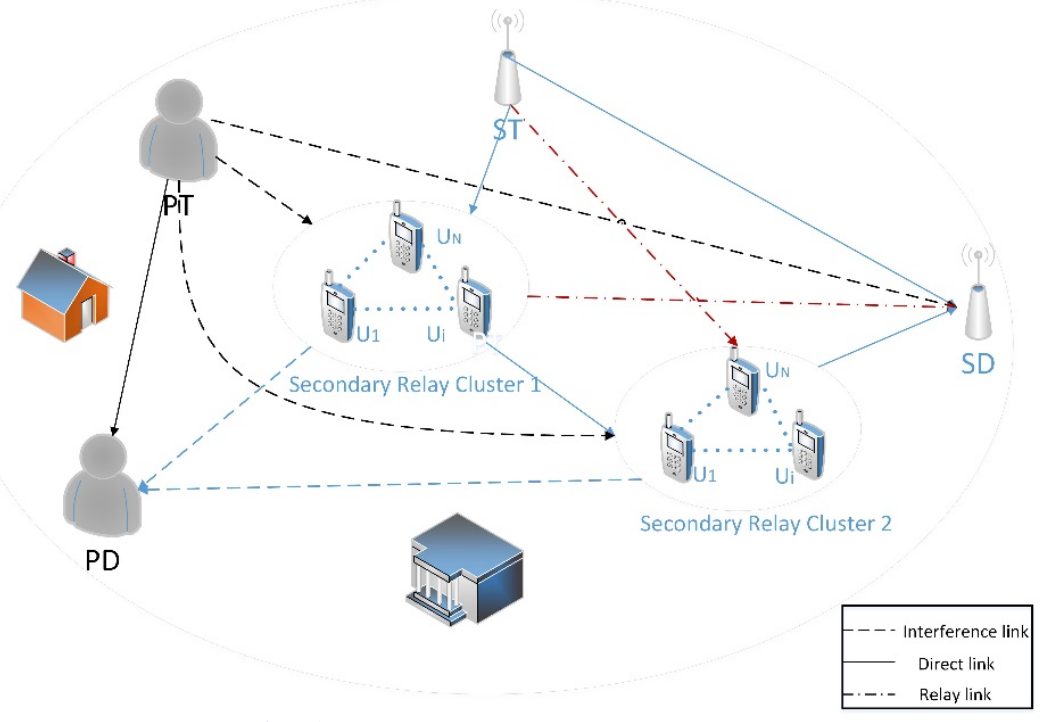

Fig. 1. System model of ICS scheme 
Relay cluster $S R_{i}$ includes $\mathrm{N}$ single-antenna half-duplex relay node and decode-and-forward protocol is considered throughout this correspondence. As can be observed from Fig. 1, primary and secondary networks would affect each other. The transmit power of ST should be limited for reducing the interference to PD in order to guarantee the QoS of primary transmissions measured by primary outage probability performance. We assume that all the channels are modeled as independent Rayleigh flat fading. We let $h_{A B}\left(A \in\left\{P T, S T, S R_{i}\right\}, B \in\left\{P D, S D, S R_{i}\right\}, A \neq B\right)$ denote fading coefficient channel from A to B with fading variance. Denote that $\mathrm{H}(H \in\{P T, S T, S R\})$ transmits $x_{H}$ to the destination with data rates $R, R_{A}$ and $R_{B}$ represent as the data rate of $\mathrm{ST}$ and PT. The transmit power of $\mathrm{H}$ is $E_{H}$ Watts/Hz. $n_{K}(K \in\{S R, S D, P D\})$ respect the additive white Gaussian noise (AWGN).

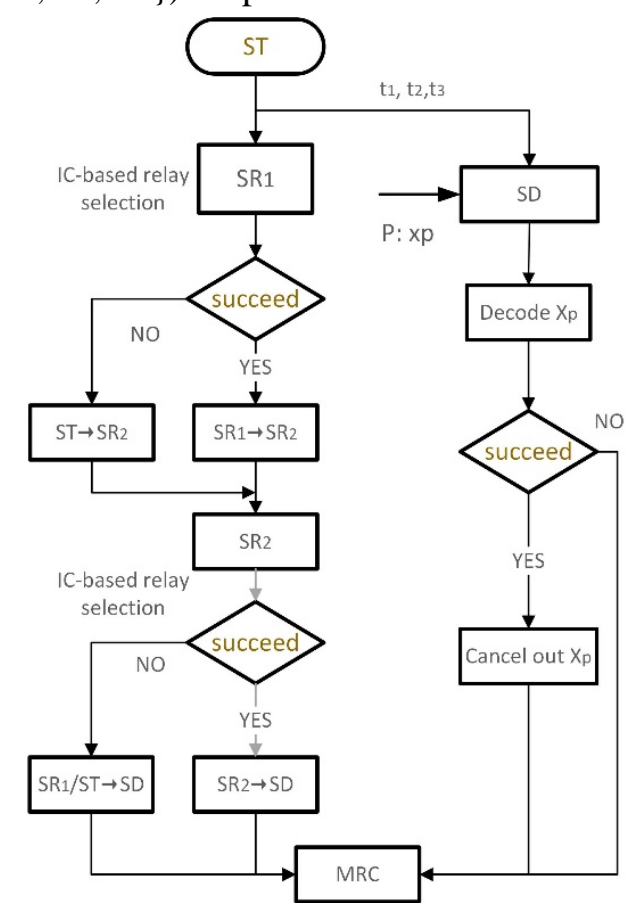

Fig. 2. Transmission process of ICS

In this paper, we assumed that ST operates in a time division multiple access fashion, where each medium access control frame consists of three consecutive transmission phases denoted by $t_{1}, t_{2}$ and $t_{3}$. As shown in Fig. 2, we propose a decoding strategy and it can be described as follows: For the first time slot, primary transmitter PT sends data $x_{P T}$ to primary destination $\mathrm{PD}$, in the meanwhile, secondary transmitter ST send data $x_{S T}$ to secondary destination SD. As is shown in Fig. 1, primary and secondary networks would affect each other. For the primary receiver, it would suffer interference from both the ST and secondary relay clusters. The secondary relays and secondary receiver are mainly interfered by PT. In order to ensure the QoS of primary transmissions, the transmit power of ST are required to be limited. Then, all relays attempt to decode the signal $x_{S T}$ by utilizing the proposed IC-based decoding technique. At first, $S R_{1}$ directly decodes $x_{S T}$ from the original received signal in $t_{1}$. If decoding fails, $S R_{1}$ will start the interference mechanism that decode $x_{P T}$ and eliminate the interference component on $x_{P T}$. In this case, SR will use the interference cancelled signal to decode $x_{S T}$ again. We make the best decoding set for the relays that utilize the improved 
interference cancellation and decode the signal successfully. At the same time, SD attempts to decode $x_{P T}$ using the received signal, and if decoding is successful, $S R_{1}$ removes the interference component of $x_{P T}$ from the original received data.

For the second time slot, if the optimal decoding set of $S R_{2}$ is not empty, then $S R_{1-b e s t}$ is selected to participate in the cooperative transmission. $S R_{2}$ uses the same interference cancellation mechanism as $S R_{1}$. Otherwise, there is no relay could decode the signal successfully in the first phase, ST delivers signal towards $S R_{2}$, while ST transmits the signal to $\mathrm{SD}$ in the second time slot.

In the third time slot, SD starts with perception, $S R_{2}$ will send data to SD if there are optimal decoding set of $S R_{2}$, simultaneously, the best relay $S R_{2-\text { best }}$ will be chosen to assist transmit data to SD. Otherwise, $S R_{1}$ or ST transmit the signal towards SD. Finally, SD try to decode $x_{P T}$ by using the received signal and removes the interference component of $x_{P T}$. Then SD performs the maximum ratio combining (MRC) technique to combine the transmission signals in three time slots and perform the final decoding.

\section{System Modeling}

The improved protocol has been described in section 2, the signals accepted at PD, $S R_{1}$ and SD during the first time slot can be respectively expressed as:

$$
\begin{aligned}
& y_{P D}(1)=\sqrt{E_{P T}} h_{P T-P D} x_{P T}(1)+\sqrt{E_{S T}} h_{S T-P D} x_{S T}+n_{P D}(1) \\
& y_{S R 1}(1)=\sqrt{E_{S T}} h_{S T-S R 1} x_{S T}+\sqrt{E_{P T}} h_{P T-S R 1} x_{P T}+n_{S R 1}(1) \\
& y_{S D}(1)=\sqrt{E_{S T}} h_{S T-S D} x_{S T}+\sqrt{E_{P T}} h_{P T-S T} x_{P T}+n_{S D}(1)
\end{aligned}
$$

Where superscript 1 means the first transmission time slot. $E_{P T}$ and $E_{S T}$ represents the transmission power of PT and ST respectively. The improved Interference elimination mechanism is used to eliminate $y_{P D}(1)$ from original signal $y_{S R 1}(1)$ and $y_{S D}(1)$ respectively. Signal after interference cancellation can be rewritten as:

$$
\begin{gathered}
y_{S R 1}^{1}(1)=\sqrt{E_{S T}} h_{S T-S R 1} x_{S T}+n_{S R 1}(1) \\
y_{S D}^{1}(1)=\sqrt{E_{S T}} h_{S T-S D} x_{S T}+n_{S D}(1)
\end{gathered}
$$

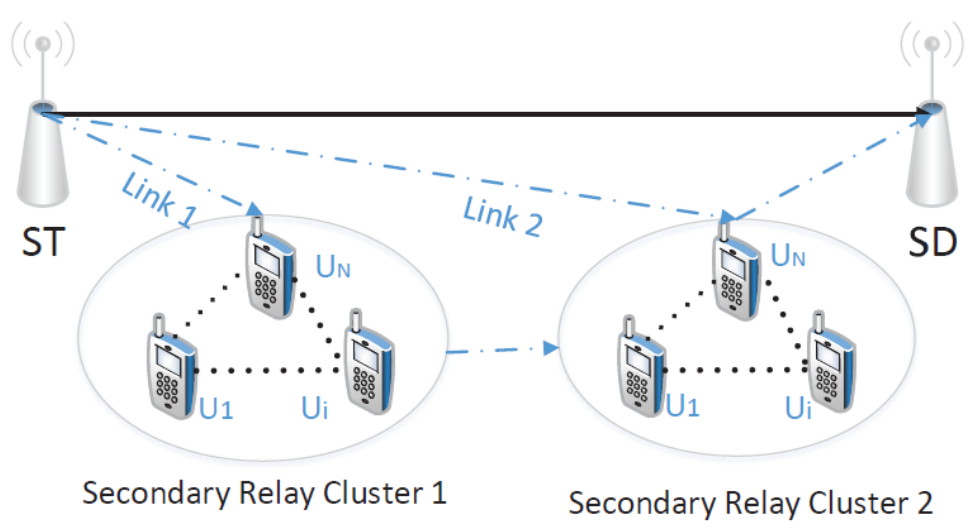

Fig. 3. Second network transmission of $t_{2}$ 
As can be shown in Fig. 3, there are two situations of transmission situation depending on whether SR1 can successfully decode the signals. In other words, two possible process rely on whether best decoding set $\Theta_{1}$ exist. We use $\Omega_{1}$ represent best decoding set and $\Xi$ represent empty set. $\Theta_{1}=\Xi_{1}$ denote that all relays fails to decode signals and $\Theta_{1}=\Omega_{1}$ indicates that more than one relay within $\Omega_{1}$ decode the signals successfully. Once the case $\Theta_{1}=\Xi_{1}$ occurs, ST will retransmit the signal to $S R_{2}$ during the second time slot. In this situation, the signal received at $\mathrm{PD}, \mathrm{SD}$ and $S R_{2}$ in $t_{2}$ can be respectively described as:

$$
\begin{aligned}
& y_{P D}(2)=\sqrt{E_{P T}} h_{P T-P D} x_{P T}(2)+\sqrt{E_{S T}} h_{S T-P D} x_{S T}+n_{P D}(2) \\
& y_{S D}(2)=\sqrt{E_{S T}} h_{S T-S D} x_{S T}+\sqrt{E_{P T}} h_{P T-S D} x_{P T}+n_{S D}(2) \\
& y_{S R 2}(2)=\sqrt{E_{S T}} h_{S T-S R 2} x_{S T}(2)+\sqrt{E_{P T}} h_{P T-S R 2} x_{P T}+n_{S R 2}(2)
\end{aligned}
$$

where the superscript 2 denotes the second transmission time slot. The received signal which adapts the interference elimination is written as:

$$
\begin{aligned}
& y_{P D}^{1}(2)=\sqrt{E_{S T}} h_{S T-S D} x_{P T}(2)+n_{S D}(2) \\
& y_{S R 2}^{1}(2)=\sqrt{E_{S T}} h_{S T-S R 2} x_{S T}(2)+n_{S R 2}(2) \\
& y_{S D}^{1}(2)=y_{S D}^{1}(2)=\sqrt{E_{S T}} h_{S T-S D} x_{S T}+n_{S D}(2)
\end{aligned}
$$

In another case, once $\Theta_{1}=\Omega_{1}$ occurs, the best relay will be chosen to assist to transmit the data to SD in $t_{2}$, the received signals of PD, SD and $S R_{2}$ in $t_{2}$ can be expressed as:

$$
\begin{aligned}
& y_{S D}(2)=\sqrt{E_{S T}} h_{S T-S D} x_{S T}+\sqrt{E_{P T}} h_{P T-S D} x_{P T}+n_{S D}(2) \\
& y_{S R 2}(2)=\sqrt{E_{S R 1}} h_{S R 1-S R 2} x_{S T}(2)+\sqrt{E_{P T}} h_{P T-S R 2} x_{P T}+n_{S R 2}(2)
\end{aligned}
$$

After Interference elimination, $\mathrm{SD}$ and $S R_{2}$ in $t_{2}$ can be expressed as:

$$
\begin{aligned}
& y_{S D}^{1}(2)=\sqrt{E_{S T}} h_{S T-S D} x_{S T}+n_{S D}(2) \\
& y_{S R 2}^{1}(2)=\sqrt{E_{S R 1}} h_{S R 1-S R 2} x_{S T}(2)+n_{S R 2}(2)
\end{aligned}
$$

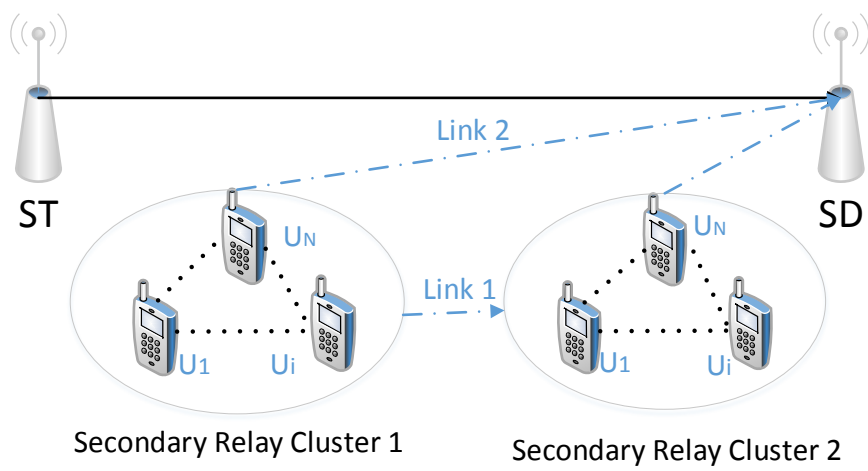

Fig. 4. Secondary network transmission of $t_{3}$

Fig. 4 shows secondary transmission during the third time slot, and there are still two situations which depends on whether $\Theta_{2}$ is an empty set. If it is an empty set, $S R_{1}$ or ST will deliver signal towards SD depending on whether $S R_{1}$ can utilize IC-based relay selection scheme to decode signals.

Case 1: This happens only $S R_{1}$ is empty set $\left(\Theta_{1}=\Xi_{1}\right)$, the signals received at PD and SD 
can be shown as:

$$
\begin{aligned}
& y_{P D}(3)=\sqrt{E_{P T}} h_{P T-P D} x_{P T}(3)+\sqrt{E_{S T}} h_{S T-P D} x_{S T}+n_{P D}(3) \\
& y_{S D}(3)=\sqrt{E_{S T}} h_{S T-S D} x_{S T}+\sqrt{E_{P T}} h_{P T-S D} x_{P T}+n_{S D}(3)
\end{aligned}
$$

Similar to the second time slot, SD get the signal after interference cancellation is shown as:

$$
y_{S D}^{1}(3)=\sqrt{E_{S T}} h_{S T-S D} x_{S T}+n_{S D}(3)
$$

Case 2: If $S R_{1}$ successfully decode the signal by taking IC-based protocol, the signal received at $\mathrm{PD}$ and $\mathrm{SD}$ can be derived as:

$$
\begin{aligned}
& y_{P D}(3)=\sqrt{E_{P T}} h_{P T-P D} x_{P T}(3)+\sqrt{E_{S R 1}} h_{S R 1-P D} x_{S R 1}+n_{P D}(3) \\
& y_{S D}(3)=\sqrt{E_{S R 1}} h_{S R 1-S D} x_{S T}+\sqrt{E_{P T}} h_{P T-S D} x_{P T}+n_{S D}(3)
\end{aligned}
$$

After interference cancellation, signal received at SD is given as follows:

$$
y_{S D}^{1}(3)=\sqrt{E_{S R 1}} h_{S R 1-S D} x_{S T}+n_{S D}(3)
$$

Eventually, SD adapts MRC to combine the signals received in $t_{1}, t_{2}$ and $t_{3}$. We assume that if the signal interference cannot be eliminated at SD, we will use the original received signal for MRC combining. Better secondary outage probability could be expected due to using the improved interference elimination scheme at both SD and PD.

In fact, we can think of this as a transmission process in multi-hop transmission. When the signal is transmitted to the Nth trunk group, the above relay selection will be performed until the signal is transmitted to the $\mathrm{N}+2$ relay. So the three-hop interference cancellation model is the simplest form of the multi-hop model.

\section{Analysis of Secondary Outage Probability}

The case corresponds to the relays in best decoding set being accessible during the first time slot, i.e.

$$
C_{S T-S R 1}=\frac{1}{3} \log _{2}\left(1+\frac{\gamma_{S T}\left|h_{S T-S R 1}\right|^{2}}{\gamma_{P T}\left|h_{P T-S R 1}\right|^{2}+1}\right)
$$

And achievable data rates can be expressed as:

$$
C_{P T-S R 1}=\frac{1}{3} \log _{2}\left(1+\frac{\gamma_{P T}\left|h_{P T-S R 1}\right|^{2}}{\gamma_{S T}\left|h_{S T-S R 1}\right|^{2}+1}\right)
$$

Notice that fading factors $\left|h_{S T-S R 1}\right|^{2}$ and $\left|h_{P T-S R 1}\right|^{2}$ are independent and follow the exponential distributions with parameters $\frac{1}{\sigma_{S T-S R 1}^{2}}$ and $\frac{1}{\sigma_{P T-S R 1}^{2}}$ respectively. As can be shown from (22), accessible data rate at SR1 can be shown as:

$$
C_{S T-S R 1}^{I C S}=\frac{1}{3} \log _{2}\left(1+\gamma_{S T}\left|h_{S T-S R 1}\right|^{2}\right)
$$

$S R_{1}$ can successfully deliver the data $x_{s}$ by direct decoding or ICS-based decoding. The occurrence probability of $S R_{1}$ successfully decode data $x_{s}$ during $t_{1}$ can be expressed as:

$$
\begin{aligned}
P_{S R 1} & =\operatorname{Pr}\left\{C_{S T-S R 1} \geq R_{A}\right\}+\operatorname{Pr}\left\{C_{S T-S R 1}<R_{A}, C_{P T-S R 1} \geq R_{B}, C_{S T-S R 1} \geq R_{A}\right\} \\
& =\left\{\begin{array}{cc}
a_{1}+a_{2}+a_{3}-a_{4} & 0<\Delta_{A} \Delta_{B}<1 \\
a_{1}+a_{3} & \Delta_{A} \Delta_{B} \geq 1
\end{array}\right.
\end{aligned}
$$


Therefore, we can utilize the table of exponential distribution to calculate the approximate detection probability, that is

$$
\begin{aligned}
& a_{1}=\frac{\gamma_{S T} \sigma_{S T-S R 1}^{2}}{\gamma_{S T} \sigma_{S T-S R 1}^{2}} \exp \left(-\frac{\Delta_{A}}{\gamma_{S T} \sigma_{S T-S R 1}^{2}}\right) \\
& a_{2}=\frac{\Delta_{A} \gamma_{P T} \sigma_{P T-S R 1}^{2}}{\Delta_{A} \gamma_{P T} \sigma_{P T-S R 1}^{2}+\gamma_{S T} \sigma_{S T-S R 1}^{2}} \exp \left(\frac{1}{\gamma_{P T} \sigma_{P T-S R 1}^{2}}-\frac{\Psi}{\gamma_{S T} \sigma_{P T-S R 1}^{2}}-\frac{\Psi}{\Delta_{A} \gamma_{P T} \sigma_{P T-S R 1}^{2}}\right) \\
& a_{3}=\frac{\gamma_{P T} \sigma_{P T-S R 1}^{2}}{\gamma_{P T} \sigma_{P T-S R 1}^{2}+\Delta_{B} \gamma_{P T} \sigma_{S T-S R 1}^{2}} \exp \left(-\frac{\Delta_{B}}{\gamma_{P T} \sigma_{P T-S R 1}^{2}}-\frac{\Delta_{A}}{\gamma_{S T} \sigma_{S T-S R 1}^{2}}-\frac{\Delta_{A} \Delta_{B}}{\gamma_{P T} \sigma_{P T-S R 1}^{2}}\right) \\
& a_{4}=\frac{\gamma_{P T} \sigma_{P T-S R 1}^{2}}{\gamma_{P T} \sigma_{P T-S R 1}^{2}+\Delta_{B} \gamma_{S T} \sigma_{S T-S R 1}^{2}} \exp \left(-\frac{\Delta_{B}}{\gamma_{P T} \sigma_{P T-S R 1}^{2}}-\frac{\Psi}{\gamma_{S T} \sigma_{S T-S R 1}^{2}}-\frac{\Psi \Delta_{B}}{\gamma_{P T} \sigma_{P T-S R 1}^{2}}\right)
\end{aligned}
$$

Where $\Delta_{A}=2^{2 R_{A}}-1, \Delta_{B}=2^{2 R_{B}}-1$ and $\Psi=\frac{\Delta_{A}\left(1+\Delta_{B}\right)}{1-\Delta_{A} \Delta_{B}}$.

During the second time slot, the detection probability of that $S R_{2}$ can successfully decode $x_{s}$ during $t_{2}$ is expressed as:

$$
P_{S R 2}=\operatorname{Pr}\left\{C_{S T-S R 2} \geq R_{A}\right\}+\operatorname{Pr}\left\{C_{S T-S R 2}<R_{A}, C_{P T-S R 2} \geq R_{B}, C_{S T-S R 2} \geq R_{A}\right\}
$$

Therefore, the occurrence probabilities of the case that both of $S R_{1}$ and $S R_{2}$ cannot decode the data correctly can be shown as:

$$
P C_{\Xi}^{I C S}=\prod_{\mathrm{i}=1}^{N} \prod_{j=1}^{M}\left(1-P_{S R_{i}}\right)\left(1-P_{S R_{j}}\right)
$$

And the probability of a link can be expressed as:

$$
P C_{\Omega}^{I C S}=\prod_{i \in \Omega_{1}}\left(P_{S R_{i}}\right) \prod_{k \in \Omega_{1}}\left(1-P_{S R_{k}}\right) \prod_{i=1}^{N}\left(1-P_{S R_{i}}\right)+\prod_{j \in \Omega_{2}}\left(P_{S R_{j}}\right) \prod_{v \in \Omega_{2}}\left(1-P_{S R_{v}}\right)
$$

Where $\overline{\Omega_{1}}$ and $\overline{\Omega_{2}}$ is the complementary set of $\Omega_{1}$ and $\Omega_{2}$ respectively. After applying the Interference elimination mechanism at SR and SD, interference from PT can be fully suppressed. From (7), the achievable data rate between PT and ST in $t_{1}$ is given as: $C_{S 0}^{P T}=\log _{2}\left(1+\frac{\Upsilon_{P T}\left|h_{P T-S D}\right|^{2}}{\Upsilon_{S T}\left|h_{S T-S D}\right|^{2}+1}\right)$.

According to section 2, if $\Theta=\Xi$ occurs, there are two situations depending on whether ST could decode the data by ICS or not. If SD fails to use ICS to decode the data, the secondary achievable data rate is shown as $C_{S 0,1}^{I C S}=\log _{2}\left(1+\frac{3 \Upsilon_{S T}\left|h_{S T-S D}\right|^{2}}{\Upsilon_{P T}\left|h_{P T-S D}\right|^{2}+1}\right)$, otherwise, the achievable data rate is $C_{S 0,2}^{I C S}=\log _{2}\left(1+3 \Upsilon_{S T}\left|h_{S T-S D}\right|^{2}\right)$. Then the outage probability of the case $\Theta=\Xi$ should be calculated as:

$$
\text { Pout }_{\Xi}=\operatorname{Pr}\left\{C_{S 0,1}^{I C S}<R_{A}, C_{S 0}^{P T}<R_{B}\right\}+\operatorname{Pr}\left\{C_{S 0,2}^{I C S}<R_{A}, C_{S 0}^{P T} \geq R_{B}\right\}
$$

For convenience, we define $X_{1}=\gamma_{S T}\left|h_{S T-S D}\right|^{2}$ and $X_{3}=\gamma_{P T}\left|h_{P T-S D}\right|^{2}$, the above formula can be written as

$$
\text { Pout }_{\Xi}=\operatorname{Pr}\left\{\frac{1}{3} \log _{2}\left(1+\frac{3 X_{1}}{X_{3}+1}\right)<R_{A}, \log _{2}\left(1+\frac{X_{3}}{X_{1}+1}\right)<R_{B}\right\}+\operatorname{Pr}\left\{\frac{1}{3} \log _{2}\left(1+3 X_{1}\right)<R_{A}, \log _{2}\left(1+\frac{X_{3}}{X_{1}+1}\right)\right\}
$$


When $\Delta_{A} \Delta_{B}<3$

Pout $_{\Xi}=\int_{0}^{\Delta_{B}} \int_{0}^{\frac{\Delta_{A}\left(x_{3}+1\right)}{3}} f\left(x_{1}\right) f\left(x_{3}\right) d x_{1} d x_{3}+\int_{\kappa}^{\Delta_{B}} \int_{\frac{\underline{x}_{3}}{\kappa}-1}^{\frac{\Delta_{A}\left(x_{3}+1\right)}{3}} f\left(x_{1}\right) f\left(x_{3}\right) d x_{1} d x_{3}+\int_{0}^{\frac{\Delta_{A}}{3}} \int_{\left(x_{1}+1\right) \Delta_{B}}^{\infty} f\left(x_{1}\right) f\left(x_{3}\right) d x_{1} d x_{3}$

When $\Delta_{A} \Delta_{B} \geq 3$

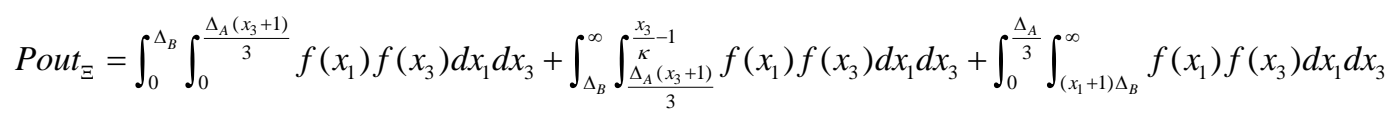

where $f\left(x_{1}\right)=\frac{1}{\gamma_{S T} \sigma_{S T-S D}^{2}} \exp \left(-\frac{x_{1}}{\gamma_{S T} \sigma_{S T-S D}^{2}}\right), f\left(x_{3}\right)=\frac{1}{\gamma_{P T} \sigma_{P T-S D}^{2}} \exp \left(-\frac{x_{3}}{\gamma_{P T} \sigma_{P T-S D}^{2}}\right), \kappa=\frac{\Delta_{B}\left(3+\Delta_{A}\right)}{3-\Delta_{A} \Delta_{B}}$.

On the other hand, when $\Theta=\Omega$ occurs, the achievable data rate between ST and SD has four possible situations as given in Table 1. For simplicity, we define $X_{1}=\gamma_{S T}\left|h_{S T-S D}\right|^{2}$, $X_{2}=\max _{i \in \Omega_{N}} \gamma_{S R_{2}}\left|h_{S R_{2}-S D}\right|^{2}, X_{3}=\gamma_{P T}\left|h_{P T-S D}\right|^{2}$. The achievable data rate between $S R_{2}$ and SD can be shown as $C_{S 0,2}^{P T}=\log _{2}\left(1+\frac{X_{3}}{X_{2}+1}\right)$.

Table 1. Secondary achievable rates of four cases

\begin{tabular}{|c|c|}
\hline Situations & Secondary achievable data rates \\
\hline ICS fails in $t_{1}, t_{2}$ and $t_{3}$ & $C_{S T-S D}^{I C S, C 4}=\frac{1}{3} \log _{2}\left(1+\frac{2 X_{1}+X_{2}}{X_{3}+1}\right)$ \\
\hline ICS succeeds in $t_{1}, t_{2}$, but fails in $t_{3}$ & $C_{S T-S D}^{I C S, C 2}=\frac{1}{3} \log _{2}\left(1+2 X_{1}+\frac{X_{2}}{X_{3}+1}\right)$ \\
\hline ICS fails in $t_{1}, t_{2}$, but succeeds in $t_{3}$ & $C_{S T-S D}^{I C S, C 3}=\frac{1}{3} \log _{2}\left(1+\frac{2 X_{1}}{X_{3}+1}+X_{2}\right)$ \\
\hline ICS succeeds in $t_{1}, t_{2}$ and $t_{3}$ & $C_{S T-S D}^{I C S, C 1}=\frac{1}{3} \log _{2}\left(1+2 X_{1}+X_{2}\right)$ \\
\hline
\end{tabular}

Therefore, the secondary outage probability conditioned on that case $\Theta=\Omega$ occurs can be calculated as:

$$
\begin{aligned}
\text { Pout }_{\Omega} & =\operatorname{Pr}\left\{C_{S T-S D}^{I C S, C 1}<R_{A}, C_{S 0}^{P T}<R_{B}, C_{S 0,2}^{P T}<R_{B}\right\} \\
& +\operatorname{Pr}\left\{C_{S T-S D}^{I C S C 2}<R_{A}, C_{S 0}^{P T} \geq R_{B}, C_{S 0,2}^{P T}<R_{B}\right\} \\
& +\operatorname{Pr}\left\{C_{S T-S D}^{I C S, C 3}<R_{A}, C_{S 0}^{P T}<R_{B}, C_{S 0,2}^{P T} \geq R_{B}\right\} \\
& +\operatorname{Pr}\left\{C_{S T-S D}^{I C S C C}<R_{A}, C_{S 0}^{P T} \geq R_{B}, C_{S 0,2}^{P T} \geq R_{B}\right\}
\end{aligned}
$$


The above formulation can be calculated as:

$$
\begin{aligned}
& \text { Pout }_{\Omega}=\operatorname{Pr}\left\{\frac{2 X_{1}+X_{2}}{X_{3}+1}<\Delta_{A}, \frac{X_{3}}{X_{1}+1}<\Delta_{B}, \frac{X_{3}}{X_{2}+1}<\Delta_{B}\right\} \\
& +\operatorname{Pr}\left\{2 X_{1}+\frac{X_{2}}{X_{3}+1}<\Delta_{A}, \frac{X_{3}}{X_{1}+1} \geq \Delta_{B}, \frac{X_{3}}{X_{2}+1}<\Delta_{B}\right\} \\
& +\operatorname{Pr}\left\{X_{2}+\frac{2 X_{1}}{X_{3}+1}<\Delta_{A}, \frac{X_{3}}{X_{1}+1}<\Delta_{B}, \frac{X_{3}}{X_{2}+1} \geq \Delta_{B}\right\} \\
& +\operatorname{Pr}\left\{2 X_{1}+X_{2}<\Delta_{A}, \frac{X_{3}}{X_{1}+1} \geq \Delta_{B}, \frac{X_{3}}{X_{2}+1} \geq \Delta_{B}\right\} \\
& =P_{1}+P_{2}+P_{3}+P_{4}
\end{aligned}
$$

According to the above integral area, when $\Delta_{A} \Delta_{B} \geq 3$, we can get the following calculation process of $P_{1}$

$$
\begin{aligned}
P_{1} & =\int_{0}^{\Delta_{B}} \int_{0}^{\frac{\Delta_{A}\left(x_{3}+1\right)}{2}} \int_{0}^{\left(x_{3}+1\right) \Delta_{A}-2 x_{1}} f\left(x_{1}\right) f\left(x_{2}\right) f\left(x_{3}\right) d x_{1} d x_{2} d x_{3} \\
& +\int_{\Delta_{B}}^{\vartheta} \int_{\frac{x_{3}}{\Delta_{B}}-1}^{\left(x_{3}+1\right) \Delta_{A}-2\left(\frac{x_{3}}{\Delta_{B}}-1\right)} \int_{\frac{x_{3}}{\Delta_{B}}-1}^{\frac{1}{2}\left[\left(x_{3}+1\right) \Delta_{A}-x_{2}\right]} f\left(x_{1}\right) f\left(x_{2}\right) f\left(x_{3}\right) d x_{1} d x_{2} d x_{3}
\end{aligned}
$$

where $\vartheta=\frac{\Delta_{A} \Delta_{B}+3 \Delta_{B}}{3-\Delta_{A} \Delta_{B}}$.

When $\Delta_{A} \Delta_{B}<3$, the calculation is as follows:

$$
\begin{aligned}
P_{1} & =\int_{0}^{\Delta_{B}} \int_{0}^{\frac{\Delta_{A}\left(x_{3}+1\right)}{2}} \int_{0}^{\left(x_{3}+1\right) \Delta_{A}-2 x_{1}} f\left(x_{1}\right) f\left(x_{2}\right) f\left(x_{3}\right) d x_{1} d x_{2} d x_{3} \\
& +\int_{\Delta_{B}}^{\infty} \int_{\frac{x_{3}}{\Delta_{B}}-1}^{\left(x_{3}+1\right) \Delta_{A}-2\left(\frac{x_{3}}{\Delta_{B}}-1\right)} \int_{\frac{x_{3}}{\Delta_{B}}-1}^{\frac{1}{2}\left(\left[x_{3}+1\right) \Delta_{A}-x_{2}\right]} f\left(x_{1}\right) f\left(x_{2}\right) f\left(x_{3}\right) d x_{1} d x_{2} d x_{3}
\end{aligned}
$$

where

$$
f\left(x_{1}\right)=\frac{1}{\gamma_{S T} \sigma_{S T-S D}^{2}} \exp \left(-\frac{x_{1}}{\gamma_{S T} \sigma_{S T-S D}^{2}}\right) \quad, \quad f\left(x_{2}\right)=\frac{1}{\gamma_{S R} \sigma_{S R-S D}^{2}} \exp \left(-\frac{x_{2}}{\gamma_{S R} \sigma_{S R-S D}^{2}}\right)
$$

$f\left(x_{3}\right)=\frac{1}{\gamma_{P T} \sigma_{P T-S D}^{2}} \exp \left(-\frac{x_{3}}{\gamma_{P T} \sigma_{P T-S D}^{2}}\right)$. The remaining probability calculation formula is similar, and the calculation process is no longer described in the text, which will be explained in detail in the appendix.

Therefore, the outage probability of system can be calculated as:

$$
\text { Pout }=\text { Pout }_{\Omega} * P C_{\Omega}^{I C S}+\text { Pout }_{\Xi} * P C_{\Xi}^{I C S}
$$

In fact, we describe the simplest model of multi-hop under the proposed interference cancellation scheme. This dual relay transfer scheme is also the selection and transfer mode of any two adjacent relays in multi-hop model. However, considering the restriction of direct link connection and system complexity, we do not specifically analyze the N-hop model. Moreover, the transmission range of dense cells is getting smaller and smaller under the 5G background, and excessively increasing the number of multi-hop is not constructive to the job of improving transmission efficiency. 


\section{Simulation and Analysis}

In this section, we evaluate the proposed information cancellation scheme and show the superiority of proposed scheme by simulations. Note that all the simulation results are for independent Rayleigh flat fading. We consume that each secondary relay clusters insist of two relays. For the sake of simplicity, we assume that $\sigma_{P T-S R}^{2}=\sigma_{P T-S D}^{2}=0.2$, $\sigma_{P T-P D}^{2}=\sigma_{S T-S D}^{2}=\sigma_{S T-S R_{1}}^{2}=\sigma_{S R_{1}-S R_{2}}^{2}=\sigma_{S R_{2}-S D}^{2}=1, \sigma_{S T-P D}^{2}=\sigma_{S R-P D}^{2}, R_{A}=0.2$ and $R_{B}=0.4$.

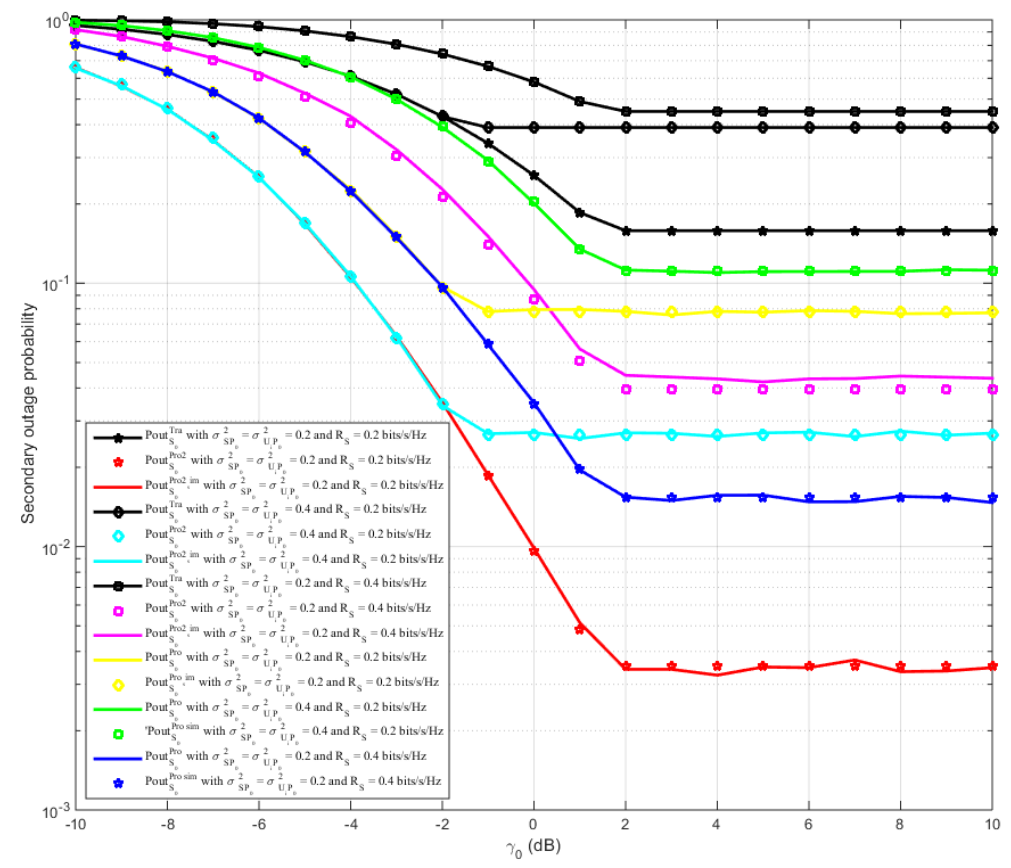

Fig. 5. Secondary outage probability versus $\gamma_{0}$

First, Fig. 5 shows the secondary outage probability versus $\gamma 0\left(E_{0} / \sigma_{0}^{2}\right)$ under different kinds of settings for traditional scheme and improved scheme. Note that Pout ${ }^{\text {Tra }}$ denotes the

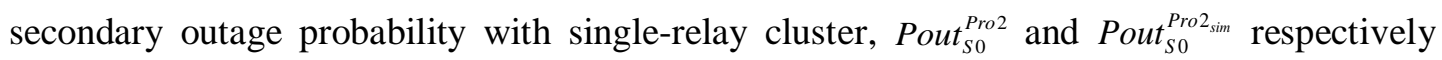
represent the theoretical and simulated values of the secondary outage probability for

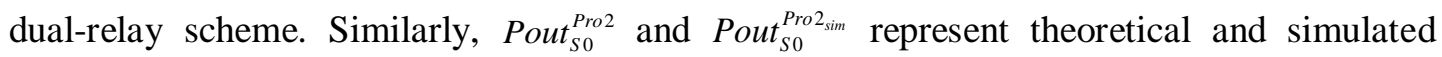
values for single-relay network. The lower corner 1 denotes $\sigma_{S T-P D}^{2}=\sigma_{S R-P D}^{2}=0.2$ and $R_{S}=0.2 \mathrm{bits} / \mathrm{s} / \mathrm{Hz}$, the lower corner 2 denotes $\sigma_{S T-P D}^{2}=\sigma_{S R-P D}^{2}=0.4$ and $R_{S}=0.2 \mathrm{bits} / \mathrm{s} / \mathrm{Hz}$, and the lower corner 3 denotes $\sigma_{S T-P D}^{2}=\sigma_{S R-P D}^{2}=0.2$ and $R_{S}=0.4 b i t s / \mathrm{s} / \mathrm{Hz}$. As expressed by following formula, dual-relay scheme adopts the same dual secondary power constraint as single-relay scheme. $T_{0}$ denotes the preset threshold ordered by primary networks. As shown in Fig. 5, proposed scheme significantly reduces the secondary outage probability compared with traditional scheme because of power constraint and interference cancellation scheme. What's more, compared with the scheme with single-relay, the improved system in this paper reduces the outage probability due to the flexible relay selection scheme. The power constraint of secondary network is the dominant factor that affects the secondary outage probability in 
low $\gamma_{0}$ area. As the $\gamma_{0}$ increases, the secondary outage probability will maintain a steady trend owning to the fact that the QoS requirement of primary network, which become the main reason for SU transmit power constraint in high $\gamma_{0}$ regime. It is important to note that, when $\gamma_{0} \leq-2$, the trend of the two curves for the improved scheme remains the same, then the downward trend of the two curves changes due to the power limitation and channel variance. As can be seen, the gap between the two curves in the same scheme in terms of outage probability increases as the channel variance increases.

$$
\begin{aligned}
& E_{S T}=\min \left(E_{S T}^{T r a}, E_{S T, T h r}\right) \\
& E_{S R}=\min \left(E_{S R}^{T r a}, E_{S R, T h r}\right)
\end{aligned}
$$

$$
\begin{aligned}
& E_{S T}^{T r a}=E_{P T} \sigma_{P T-P D}^{2} \max \left(\frac{1}{1-T_{0}} e^{\frac{\Delta_{B}}{\gamma \sigma_{P T-P D}^{2}}}-1,0\right) /\left(\Delta_{B} \sigma_{S T-P D}^{2}\right) \\
& E_{S R}^{T r a}=E_{P T} \sigma_{P T-P D}^{2} \max \left(\frac{1}{1-T_{0}} e^{\frac{\Delta_{B}}{\gamma \sigma_{P T-P D}}}-1,0\right) /\left(\Delta_{B} \sigma_{S R-P D}^{2}\right)
\end{aligned}
$$

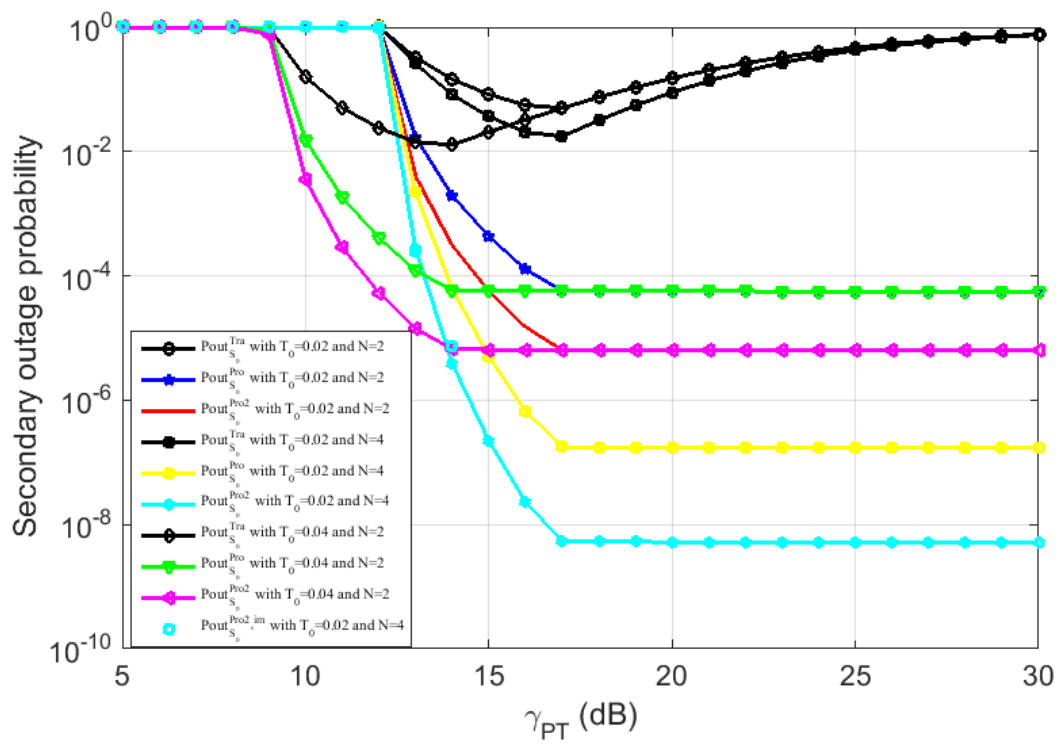

Fig. 6. Secondary outage probability versus $\gamma_{P T}$

In Fig. 6 we study the impact of $\gamma_{P T}$ on the secondary outage probability by comparing the conventional scheme and improved scheme. $T_{0}$ denotes the preset threshold ordered by primary networks. It is noteworthy that upon raising the number of $\gamma_{P T}$ up to about 18, the system performance degrades as expected in terms of secondary outage probability due to interference from PT. However, comparing with conventional scheme, the decline of secondary outage probability of improved scheme is more obvious because of the IC-based relay selection scheme. Concerning the multi-hop based on ICS, we compared the improved three-hop scheme with two-hop scheme, as can be shown in the Fig. 6, the three-hop scheme can achieve lower secondary outage probability. Note that more flexible IC-based relay selection scheme could ensure the reliability of secondary transmission link and improve the effect of secondary transmission in the system model. In low $\gamma_{P T}$ regions, the secondary 
outage probability is equal to 1 , which due to the fact that the secondary transmission are not permitted in order to ensure the PU QoS when $\gamma_{P T}$ is low. The higher is $\gamma_{P T}$, the lower is the secondary outage probability, due to the fact that more available transmit power is allowed in secondary system. Moreover, in order to evaluate the impact of numbers of relays on the secondary system performance, we calculate the system performance in terms of secondary outage probability achieved by traditional scheme, two-hop relay scheme and improved ICS scheme. As relay number increases in each relay cluster, each mechanism will have significant reduction in the secondary outage probability.

If the SU receiver can reduce the interference close to zero, we can make the definition of the generalized diversity gain as an asymptotic ratio of secondary outage probability to the interference gain as follows:

$$
d^{\text {pro }}=\lim _{\sigma_{S T-P D}^{2} \rightarrow 0} \log \left(\lim _{\sigma_{S R-P D \rightarrow \infty}^{2}} \text { Pout } t_{S D}^{\text {pro }}\right) / \log \left(\sigma_{S T-P D}^{2}\right)
$$

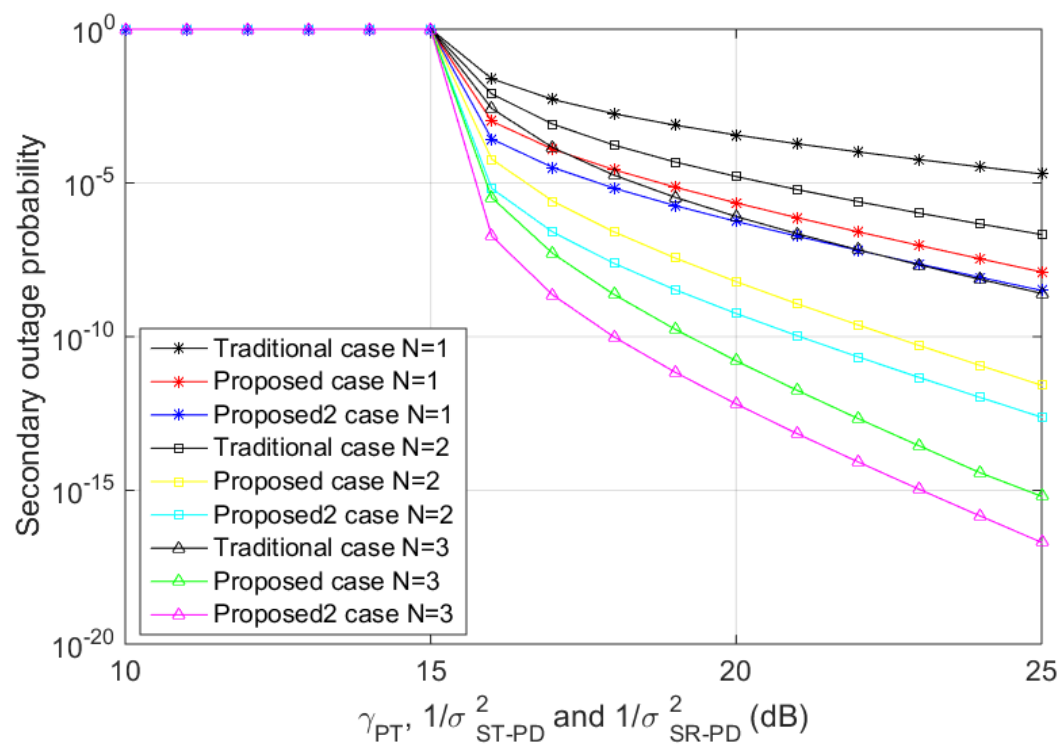

Fig. 7. Illustration of generalized diversity gain

The proposed analysis of the exact generalized diversity gain of the underlying protocol is beyond the scope of this article. Fig. 7 shows the generalized diversity gain by simulation for conventional and improved scheme, where power constraint imposed by secondary networks will be removed. Fig. 7 shows that the underlying improved protocol achieves a higher degree of diversity than the traditional cases owing to the fact that the interfering link from the primary transmitter to the secondary destination can utilize additional diversity gain by using ICS on the secondary users.

\section{Conclusion}

In the final analysis, we focus on three-hop cooperative system and propose IC-based transmission protocol for underlay CRNs, where a flexible IC-based scheme is utilized at both the secondary relays and the secondary destination. The proposed IC-based transmission scheme indeed improves the secondary relay transmission efficiency and reduces the possibility of interruption drastically by adapting. What's more, secondary users transmits 
signals under dual secondary power limitations which is restricted by both primary and secondary systems. Under the stringent power constraint, the improved system can ensure both the QoS requirement of primary users and the reduction of secondary outage probability. We evaluate the secondary transmission system in terms of secondary outage probability and derive the closed-form expression over Rayleigh fading channels. As simulation results show, even with the increased hop number of relays, the secondary outage probability is still reduced due to the flexibility of the improved protocol. Furthermore, the proposed protocol can be easily extended to multi-hop transmission system where multiple relays assisted secondary transmissions would almost eliminate the interference from primary users.

\section{APPENDIX}

When $\Delta_{A} \Delta_{B} \geq 1$, we can get the following calculation process of $P_{2}$

$$
P 2=\int_{\Delta_{B}}^{\tau} \int_{0}^{\frac{x_{3}}{\Delta_{B}}-1} \int_{0}^{\left(x_{3}+1\right)\left(\Delta_{A}-2 x_{1}\right)} f\left(x_{1}\right) f\left(x_{2}\right) f\left(x_{3}\right) d x_{1} d x_{2} d x_{3}+\int_{\tau}^{\infty} \int_{\frac{x_{3}}{\Delta_{B}}-1}^{\left(x_{3}+1\right) \Delta_{A}} \int_{0}^{\frac{1}{2}\left(\Delta_{A}-\frac{x_{2}}{x_{3}+1}\right)} f\left(x_{1}\right) f\left(x_{2}\right) f\left(x_{3}\right) d x_{1} d x_{2} d x_{3}
$$

When $\Delta_{A} \Delta_{B}<1$, the calculation is as follows:

$$
P 2=\int_{\Delta_{B}}^{\tau} \int_{0}^{\frac{x_{3}}{\Delta_{B}}-1} \int_{0}^{\left(x_{3}+1\right)\left(\Delta_{A}-2 x_{1}\right)} f\left(x_{1}\right) f\left(x_{2}\right) f\left(x_{3}\right) d x_{1} d x_{2} d x_{3}+\int_{\tau}^{\bar{\sigma}} \int_{\frac{x_{3}}{\Delta_{B}}-1}^{\left(x_{3}+1\right) \Delta_{A}} \int_{0}^{\frac{1}{2}\left(\Delta_{A}-\frac{x_{2}}{x_{3}+1}\right)} f\left(x_{1}\right) f\left(x_{2}\right) f\left(x_{3}\right) d x_{1} d x_{2} d x_{3}
$$

where $\tau=\frac{\left(2 \Delta_{B}+\Delta_{A} \Delta_{B}-3\right)+\sqrt{\left(2 \Delta_{B}+\Delta_{A} \Delta_{B}-3\right)^{2}+8 \Delta_{B}\left(3+\Delta_{A}\right)}}{4}, \varpi=\frac{\Delta_{B}\left(1+\Delta_{A}\right)}{1-\Delta_{A} \Delta_{B}}$.

$P_{3}$ can be calculated as:

When $\Delta_{A} \Delta_{B} \geq 2$

$$
P 3=\int_{\Delta_{B}}^{\varsigma} \int_{0}^{\frac{x_{3}}{\Delta_{B}}-1} \int_{\frac{x_{3}}{\Delta_{B}}-1}^{\frac{\left(\Delta_{A}-x_{2}\right)\left(x_{3}+1\right)}{2}} f\left(x_{1}\right) f\left(x_{2}\right) f\left(x_{3}\right) d x_{1} d x_{2} d x_{3}+\int_{\varsigma}^{\infty} \int_{\frac{x_{3}}{\Delta_{B}}-1}^{\Delta_{A}-\frac{2 x_{1}}{x_{3}+1}} \int_{0}^{\Delta_{A}--\frac{2 x_{1}}{x_{3}+1}} f\left(x_{1}\right) f\left(x_{2}\right) f\left(x_{3}\right) d x_{1} d x_{2} d x_{3}
$$

When $\Delta_{A} \Delta_{B}<2$

$$
P 3=\int_{\Delta_{B}}^{\varsigma} \int_{0}^{\frac{x_{3}}{\Delta_{B}}-1} \int_{\frac{x_{3}}{\Delta_{B}}-1}^{\frac{\left(\Delta_{A}-x_{2}\right)\left(x_{3}+1\right)}{2}} f\left(x_{1}\right) f\left(x_{2}\right) f\left(x_{3}\right) d x_{1} d x_{2} d x_{3}+\int_{\varsigma}^{\chi} \int_{\frac{x_{3}}{\Delta_{B}}-1}^{\Delta_{A}-\frac{2 x_{1}}{x_{3}+1}} \int_{0}^{\Delta_{A}-\frac{2 x_{1}}{x_{3}+1}} f\left(x_{1}\right) f\left(x_{2}\right) f\left(x_{3}\right) d x_{1} d x_{2} d x_{3}
$$

where $\chi=\frac{-\left(3-\Delta_{A} \Delta_{B}-\Delta_{B}+\sqrt{\left(3-\Delta_{A} \Delta_{B}-\Delta_{B}\right)^{2}+4\left(\Delta_{A} \Delta_{B}+3 \Delta_{B}\right)}\right)}{2}, \varsigma=\frac{\Delta_{B}\left(2+\Delta_{A}\right)}{2-\Delta_{A} \Delta_{B}}$.

P4 can be calculated as: 


$$
\begin{aligned}
P 4 & =\int_{\Delta_{B}}^{\Delta_{B}\left(\frac{\Delta_{A}}{3}+1\right)} \int_{0}^{\frac{x_{3}}{\Delta_{B}}-1} \int_{0}^{\frac{x_{3}}{\Delta_{B}}-1} f\left(x_{1}\right) f\left(x_{2}\right) f\left(x_{3}\right) d x_{1} d x_{2} d x_{3} \\
& +\int_{\Delta_{B}}^{\Delta_{B}\left(\frac{\left.\Delta_{A}+1\right)}{3}+1\right)}\left[\int_{0}^{\frac{x_{3}}{\Delta_{B}}-1} \int_{0}^{\frac{1}{2}\left[a-\left(\frac{x_{3}}{\Delta_{B}}\right)\right]} f\left(x_{1}\right) f\left(x_{2}\right) d x_{1} d x_{2}+\int_{\Delta_{A}-2\left(\frac{x_{3}}{\Delta_{B}}-1\right)}^{\frac{x_{3}}{\Delta_{B}}-1} \int_{0}^{\frac{\Delta_{A}-x_{2}}{2}} f\left(x_{1}\right) f\left(x_{2}\right) d x_{1} d x_{2}\right] d x_{3} \\
& +\int_{\Delta_{B}\left(\Delta_{A}+1\right)}^{\infty} \int_{0}^{\frac{\Delta_{A}}{2}} \int_{0}^{\Delta_{A}-2 x_{1}} f\left(x_{1}\right) f\left(x_{2}\right) f\left(x_{3}\right) d x_{1} d x_{2} d x_{3}
\end{aligned}
$$

\section{References}

[1] Jeffrey G. Andrews, Stefano Buzzi, Wan Choi, "What Will 5G Be?,” IEEE Journal on Selected Areas in Communications, Vol.32, No.6, pp, 1065-1082, 2014. Article (CrossRef Link).

[2] Afif Osseiran, Federico Boccardi, Volker Braun, "Scenarios for 5G Mobile and Wireless Communications:The Vision of the METIS Project," IEEE Journals and Magazines, Vol.52, No.5, pp.26-35, 2014. Article (CrossRef Link).

[3] Panagiotis Demestichas, Andreas Georgakopoulos, Dimitrios Karvounas, "5G on the Horizon," IEEE Vehicular Technology Magazine, Vol.8, No.3, pp.47-53, jul, 2013. Article (CrossRef Link).

[4] Bin Shen, Zhenzhu Lei, Xiaoge Huang, "An Interference Contribution Rate Based Small Cells On/Off Switching Algorithm for 5G Dense Heterogeneous Networks," IEEE Access, Vol.6, pp.29757-29769, 2018. Article (CrossRef Link).

[5] Victor Fernandez-Lopez, Klaus I. Pedersen, Jens Steiner, Beatriz Soret, Preben Mogensen, "Interference Management with Successive Cancellation for Dense Small Cell Networks," in Proc. of 2016 IEEE 83rd Vehicular Technology Conference (VTC Spring), pp.1-5, 2016. Article (CrossRef Link).

[6] Ramez Askar, Abdulsalam Hamdan, Wilhelm Keusgen, Thomas Haustein, "Analysis of Utilizing Lossless Networks for Self-Interference Cancellation Purpose," in Proc. of 2018 IEEE Wireless Communications and Networking Conference (WCNC), pp.1-6, 2018. Article (CrossRef Link).

[7] Naga Bhushan, Junyi Li, Durga Malladi, Rob Gilmore, Dean Brenner, Aleksandar Damnjanovic, Ravi Teja Sukhavasi, Chirag Patel, Stefan Geirhofer, "Network Densification: The Dominant Theme for Wireless Evolution into 5G," IEEE Communications Magazine, Vol.52, No. 2, pp.82-89, 2014. Article (CrossRef Link).

[8] Cordeiro, C., K. Challapali, and D. Birru, "IEEE 802.22: An introduction to the first wireless standard based on cognitive radios," Journal of Communications, Vol. 1, No. 1, Apr, 2006. Article (CrossRef Link).

[9] Kolodzy, P., "Spectrum policy task force: Findings and recommendations," in Proc. of Internaitonal Symposium on Advanced Radio Technologies (ISART), Mar., 2003. Article (CrossRef Link).

[10] S. Haykin, "Cognitive radio: brain-empowered wireless communications," IEEE journal, vol.23, No.2, Feb, 2005. Article (CrossRef Link).

[11] Xuemin Hong, Jing Wang, Cheng-Xiang Wang, Jianghong Shi, "Cognitive Radio in 5G: A Perspective on Energy-Spectral Efficiency Trade-off,” IEEE Communications Magazine, Vol.52, No.7, pp.46-53, 2014. Article (CrossRef Link).

[12] Tevfik Yucek, Huseyin Arslan, "A survey of spectrum sensing algorithms for cognitive radio applications," IEEE Communications Surveys and Tutorials, vol.11, No.1, pp116-130, March, 2009. Article (CrossRef Link).

[13] Qing Zhao, Ananthram Swami, "A Survey of Dynamic Spectrum Access: Signal Processing and Networking Perspectives," in Proc. of 2007 IEEE International Conference on Acoustics, Speech and Signal Processing - ICASSP '07, pp.1349-1352, 2007. Article (CrossRef Link).

[14] Qing Zhao, Brian M. Sadler, “A Survey of Dynamic Spectrum Access,” IEEE Signal Processing Magazine, Vol.24, No.3, May, 2007. 
[15] Wafa Hedhly, Osama Amin, Mohamed-Slim Alouini, "Interweave Cognitive Radio with Improper Gaussian Signaling,” in Proc. of GLOBECOM 2017 - 2017 IEEE Global Communications Conference, pp.1-6,2017. Article (CrossRef Link).

[16] Vikram Chandrasekhar, Jeffrey G. Andrews, Tarik Muharemovic, "Power control in two-tier femtocell networks,” IEEE TRANS, vol.8, No.8, pp. 4316-4328, Aug.2009. Article (CrossRef Link).

[17] Y. Zou, Y. D. Yao, and B. Zheng, "A cooperative sensing based cognitive relay transmission scheme without a dedicated sensing relay channel in cognitive radio networks," IEEE Transactions on Signal Processing, vol. 59, no. 2, pp. 854-859, Feb, 2011.

[18] Antonio G. Marques, Luis M. Lopez-Ramos, Georgios B. Giannakis, Javier Ramos, "Resource Allocation for Interweave and Underlay CRs Under Probability-of-Interference Constraints," IEEE Journal on Selected Areas in Communications, Vol.30, No.10, pp.1922-1933, Oct, 2012. Article (CrossRef Link)

[19] Jemin Lee, Hano Wang, Jeffrey G. Andrews, “Outage Probability of Cognitive Relay Networks with Interference Constraints,” IEEE TRANS, Vol.10, No.2, pp.390-395, Feb, 2011. Article (CrossRef Link).

[20] Zeyang Dai, Jian Liu , and Keping Long, "Cooperative Relaying with Interference Cancellation for Secondary Spectrum Access,” KSII TRANS, vol.6, pp.2455-2472, 2012. Article (CrossRef Link).

[21] N. Devroye, P. Mitran, V. Tarok, "Cognitive multiple access networks," in Proc. of IEEE Conferences, pp.57-61, 2005. Article (CrossRef Link).

[22] Ioanna Kakalou, Kostas E. Psannis, “A broadcast protocol in Multi-Hop Cognitive Radio Ad Hoc Networks with guarantees,” in Proc. of IEEE, pp.1-7, 2017. Article (CrossRef Link).

[23] Jinlin Peng, Hao Yue, Kaiping Xue, "Energy-Aware Scheduling for Multi-Hop Cognitive Radio Networks," IEEE Transactions on Cognitive Communications and Networking, Vol.2, No. 4, pp.397-410, Oct, 2016. Article (CrossRef Link).

[24] Ilgin Safak, Ali Ozgur Yilmaz, "Error Rate Analysis of GF(q) Network Coded Detect-and-Forward Wireless Relay Networks Using Equivalent Relay Channel Models,” IEEE Trans, vol.12, No.8, pp. 3908-3919, Aug, 2013. Article (CrossRef Link).

[25] Alexander Sayenko, Mikhail Zolotukhin, "Simulation and Performance Analysis of Frame Structures for Multi-hop Relay Systems,” in Proc. of 2017 IEEE Conference on Standards for Communications and Networking (CSCN), pp.144-150, 2017. Article (CrossRef Link).

[26] Hari Krishna Boddapati, Manav R. Bhatnagar, Shankar Prakriya, “Ad-Hoc Relay Selection Protocols for Multi-Hop Underlay Cognitive Radio Networks,” in Proc. of IEEE Globecom workshops, pp.1-6, Dec, 2016. Article (CrossRef Link).

[27] Daquan Feng, Chenzi Jiang, Gubong Lim, “A survey of energy-efficient wireless communications,” IEEE Communications Society, Vol.15, No.1, pp.167-178, Feb, 2012. Article (CrossRef Link).

[28] Hari Krishna Boddapati, Manav R. Bhatnagar , Shankar Prakriya, "Performance Analysis of Cluster-Based Multi-Hop Underlay CRNs Using Max-Link-Selection Protocol,” IEEE TRANS, Vol .4, no, 1, pp. 15-29, Oct, 2017. Article (CrossRef Link).

[29] Jian Chen, Lu Lv, Yiyang Liu, "Energy efficient relay selection and power allocation for cooperative cognitive radio networks,” IET, Vol.9, No. 13, pp.1661-1668, Aug, 2015. Article (CrossRef Link).

[30] Dongqing Xie, Xiazhi Lai, Xianfu Lei, “Cognitive Multiuser Energy Harvesting Decode-and-Forward Relaying System with Direct Links,” IEEE Access, Vol. 6, pp. 5596-5606, Nov, 2017. Article (CrossRef Link).

[31] Jing Zhang, Xi Yang, Qi Yao, Xiaohu Ge, Minho Jo, Guoqiang Mao, "Cooperative Energy Efficiency Modeling and Performance Analysis in Co-Channel Interference Cellular Networks," The Computer Journal, Vol.56, No.8, pp.1010-1019, August 2013. Article (CrossRef Link).

[32] Y. Jiang, W. Ding, Z. Xi, N. Zhao and M. Wu, "Interference cancellation for two interferences with single co-site interference cancellation system," in Proc. of 2017 IEEE 17th International Conference on Communication Technology (ICCT), Chengdu, pp. 10-13, 2017.

Article (CrossRef Link) 


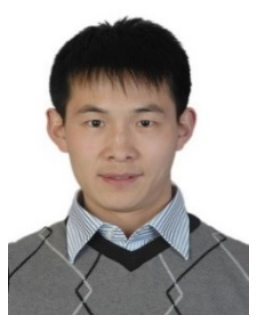

Yinghua Zhang is currently working toward the Ph.D. degree of Communication and Information Systems in School of Computer and Communication Engineering, University of Science and Technology Beijing, China. He also serves as a researcher in Dawning Information Industry (Beijing) Corp., Ltd. His current research interest is includes cognitive radio and next generation wireless communication systems. E-mail: 82774807@qq.com

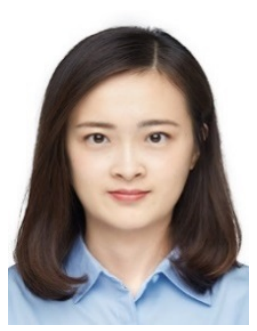

Lei Wang is currently a Master student of Communication and Information Systems in School of Computer and Communication Engineering, University of Science and Technology Beijing, China. Her current research interests include multi-hop wireless communication, cognitive radio and next generation wireless communication systems.

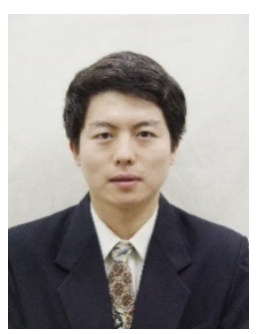

Jian Liu received his B.S. degree in Automatic Control Theory and Applications from Shandong University, China in 2000, and the Ph.D. degree in School of Information Science and Engineering from Shandong University in 2008. He is currently an associate professor in University of Science and Technology Beijing, China. His research interests include cognitive radio networks, mobile mesh networks, and key technology of next generation wireless communication systems. $\mathrm{He}$ is an IEEE member since 2009. E-mail: liujian@ustb.edu.cn

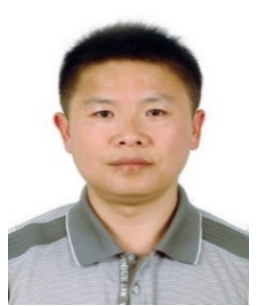

Yunfeng Peng received the Ph.D. degree in Communication engineering from Shanghai Jiao Tong University in 2007. He has been a faculty member in School of Computer and Communication Engineering, University of Science and Technology Beijing, China. His current research is Optical communication. E-mail: pengyf@ustb.edu.cn. 